\title{
Repair of giant inguino-scrotal hernia with loss of domain using minimally invasive anterior component separation technique combined with Lichtenstein tension-free mesh hernioplasty
}

\author{
Pooja Sewalia*, Avneet S. Chawla, Lirangla T Sangtam, Himaja Mandalapu, \\ Hemant Kumar, Avneet Kaur, Anshul Bansal
}

Department of General Surgery, VMMC and Safdarjung Hospital, Delhi, India

Received: 20 October 2020

Accepted: 09 December 2020

*Correspondence:

Dr. Pooja Sewalia,

E-mail: poojasewaliaa@gmail.com

Copyright: (c) the author(s), publisher and licensee Medip Academy. This is an open-access article distributed under the terms of the Creative Commons Attribution Non-Commercial License, which permits unrestricted non-commercial use, distribution, and reproduction in any medium, provided the original work is properly cited.

\begin{abstract}
Inguinal hernia repairs are most commonly performed surgical procedures across the world. Lichtenstein's tension free technique of open hernioplasty is the gold standard technique, while laparoscopic techniques gained popularity over recent decade. Giant inguinal hernias are rare. Giant inguinal hernia extends below the midpoint of the inner thigh, in the standing position. These are long standing conditions and at presentation years of herniation or even decades. We report a patient of 65 years of age presented with type-II left sided giant inguinoscrotal hernia from last 10 years with loss of domain. Contrast enhanced computed tomography (CECT) revealed, omentum and ileal loops with mesentry as contained in hernia sac, which was repaired by minimally invasive anterior component separation technique to increase the intra-abdominal volume followed by omentectomy and Lichtenstein mesh hernioplasty without any complications. He recovered uneventfully. Surgical management of giant inguinal hernia is significantly more challenging and unusual because of 'loss of domain' and returning herniated viscera into the empty abdominal cavity forcefully can lead to high intra-abdominal pressure, recurrence or abdominal compartment syndrome. There are several repair techniques in literatures such as resection of contents and increased intra-abdominal volume increasing procedures but there is no standard protocol or surgical procedure for the management of giant hernias. We describe a technique which is relatively simple, less expensive and less invasive used for type II unilateral giant inguinoscrotal hernia with loss of domain in patient with co-morbidities.
\end{abstract}

Keywords: Giant inguinoscrotal hernia, Inguinal hernia, Loss of domain, Anterior component separation

\section{INTRODUCTION}

Inguinal hernia is one of the most common surgical diseases encountered by general surgeons. Groin hernia repair surgeries were performed in the ancient times also. ${ }^{1}$ Giant inguinoscrotal hernias are uncommon and are defined as hernias extending below the midpoint of the inner thigh in standing position or an irreducible hernia present for over 10 years measuring at least $30 \mathrm{~cm}$ on antero-posterior diameter or $50 \mathrm{~cm}$ on latero-lateral diameter. ${ }^{2,3}$ Giant inguinal hernias significantly affect the patient's quality of life including difficulty in micturition, ambulation, bowel obstruction, scrotal skin ulceration, excoriation, etc. it doesn't only affects the patient physically but it also affects the mental health of the patient. Poor sexual wellbeing may be a significant source of anxiety and stress in the patient. Thus, surgeries of giant inguinal hernias are often challenging and unusual. These kinds of hernias carry risk of wound dehiscence, recurrence, abdominal compartment syndrome and potentially fatal intra-abdominal hypertension, if forced closure is done. ${ }^{4}$ Also, raised intra-abdominal and intra thoracic pressure may precipitate cardiac and respiratory failure. ${ }^{5}$ Many treatments are developed till now but none 
of them has adopted as standard procedure for this rare disease. All the major techniques mainly focused to address the "loss of domain" including debulking of abdominal contents by omentectomy, total colectomy, right hemicolectomy and small bowel resection. ${ }^{6,7}$ Selection of a particular procedure is very difficult and decision is usually made intra-operatively. These all techniques involve resection of abdominal viscera and limited by morbidity and mortality in the patient.

This article is an examination of a rare case of giant inguinal hernia containing greater omentum, ileal loops and mesentry with loss of domain. This was corrected by Lichtenstein open mesh hernioplasty in adjunct with minimally invasive anterior component separation technique to increase the intra-abdominal volume.

\section{CASE REPORT}

A 65 year old male presented with left sided inguinoscrotal swelling since 10 years, insidious onset, gradually progressive, recently became irreducible and reached below to the level of mid-thigh. Quality of life of the patient has been compromised and he was not able to perform activities of daily living normally due to constant dragging type of pain in his scrotum. There was history of chronic cough and constipation since 2 years. He was known chronic smoker and tobacco chewer. Patient was also known case of diabetes, hypertension, chronic obstructive pulmonary disease (COPD) and had history of pulmonary tuberculosis 1 year back for which he completed course of anti-tubercular treatment for 6 month. No history of vomiting, weight loss, trauma, difficulty in micturition was obtained. On general examination, blood pressure (BP) - 154/95 and pulse rate (PR) - 88/min with body mass index (BMI) of 29.1 All the blood investigations were normal except blood sugar levels which were deranged. Random blood sugar (RBS) was around $355 \mathrm{mg} / \mathrm{dl}$. Urine examination was done which was negative for urine ketones. On chest examination bilateral rhonchi were present. Scrotal examination revealed an oval shaped massive left inguino-scrotal swelling extending below the mid-thigh in standing position, partially reducible, doughy in consistency, no cough impulse, no rise in temperature, non-tender, not able to get above the swelling. There was no evidence of inflammation, excoriation, or ulceration of the scrotal skin Chest X-ray revealed miliary opacities in bilateral lungs with left sided tracheal shift. Pulmonary function tests (PFT) showed severe restrictive lung disease pattern. Contrast enhanced computed tomography (CECT) chest reported left lobe atelectasis with adjacent pleural thickening and left sided pleural effusion of $1.2 \mathrm{~cm}$. CECT abdomen showed large hernia sac containing omentum, ileal loops with its mesentry. Tanaka index was around $52.5 \%$.

Patient was posted for elective hernia repair, asked to stopped smoking and tobacco chewing 1 month before the surgery and started with formoterol fumarate and budesonide inhaler $200 \mathrm{mg} 2$ puffs twice a day. He was also started on tablet amlodipine $5 \mathrm{mg}$ once daily (OD) and oral hypoglycemic agents (OHA) with regular blood sugar and blood pressure monitoring along with diabetic and low salt diet. He was admitted 3 days before the surgery for work-up and optimization. One day before the surgery patient complaints of fever and breathlessness. Symptomatic treatment was given. Patient came out to be positive for COVID-19. Hence surgery was postponed and patient was shifted to corona wards and was followed up on regular basis by our team. After 25 days when he tested negative for COVID-19, he was again planned for elective surgery. Patient was not fit for general anesthesia because of poor chest condition which became worse due to COVID-19. Chest physiotherapy was started along with 6 hourly nebulization, bowel preparation was done, the day before surgery. During anesthetic assessment surgery was decided to be done under combined epidural and spinal anesthesia. Bladder catheterization was done after anesthesia. A small transverse incision was made $1 \mathrm{~cm}$ lateral to the semilunaris line in inguinal region of right side. The tip of plastic suction handle without suction, was inserted through the opening; in the avascular plane between the internal and external oblique aponeurosis at their junction with the rectus sheath, the tip was advanced from pubis inferiorly towards the costal margin cranially. A subaponeurotic access tunnel was created digitally lateral to the whole length of linea semilunaris bilaterally. Through the subaponeurotic access tunnel, the external aponeurosis was incised vertically $1.5-2 \mathrm{~cm}$ lateral to the linea semilunaris, over the plastic suction handle placed in the avascular plane between external and internal oblique aponeurosis with the help of laparoscopic diathermy hook. Then after, left inguinal incision was given tunnel was made on left side and same procedure was repeated. An indirect hernia sac was present which was carefully dissected separating the cord structures. The sac was opened and contents found to be omentum, ileal loops with its mesentry. Omentectomy along with adhesioloysis was done. Contents were successfully reduced back to the abdominal cavity through wide deep ring without any change. Intra-abdominal pressure was measured and confirmed by intravesicular technique.

Lichtenstein's tension free technique was performed with polypropylene mesh. Hemostasis was checked. Drain was placed in scrotum and wound closure was done in layers. Post operatively, patient was given scrotal support and epidural analgesia.

Postoperatively, the patient was managed according to the enhanced recovery after surgery (ERAS) protocol guidelines. Recovery was satisfactory with early return of bowel movements. Vacuum drain had $20 \mathrm{ml}$ of serosanguinous discharge which gradually decreases and eventually became nil on post-operative day 3 after which drain was removed. He had no significant post-operative complications and was discharged on post-operative day 8 after suture removal and followed up in the outpatient department (OPD) on regular basis. 


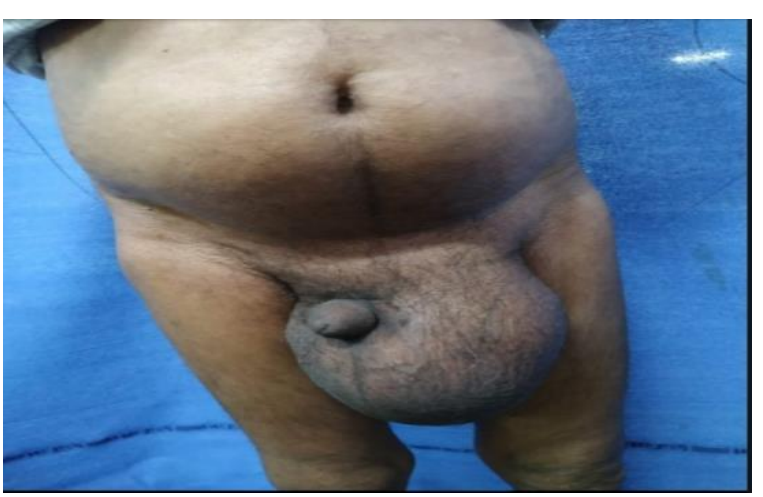

Figure 1: Left sided type II giant inguinoscrotal hernia.

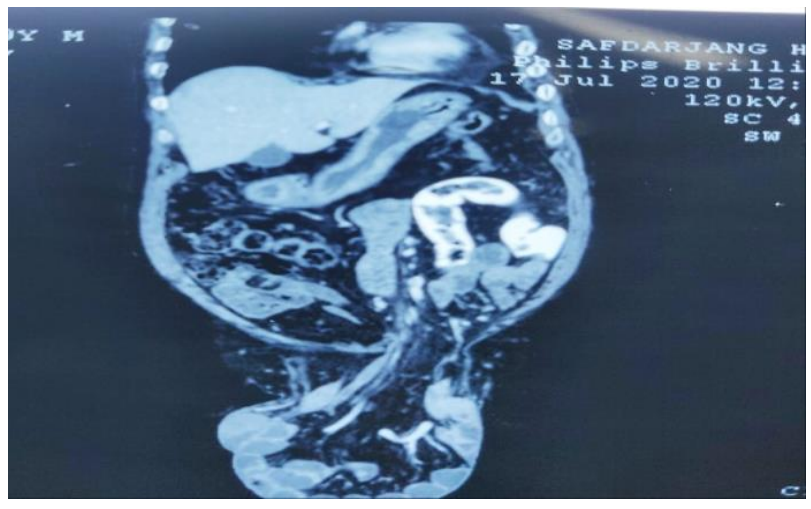

Figure 2: CECT of abdomen and pelvis.

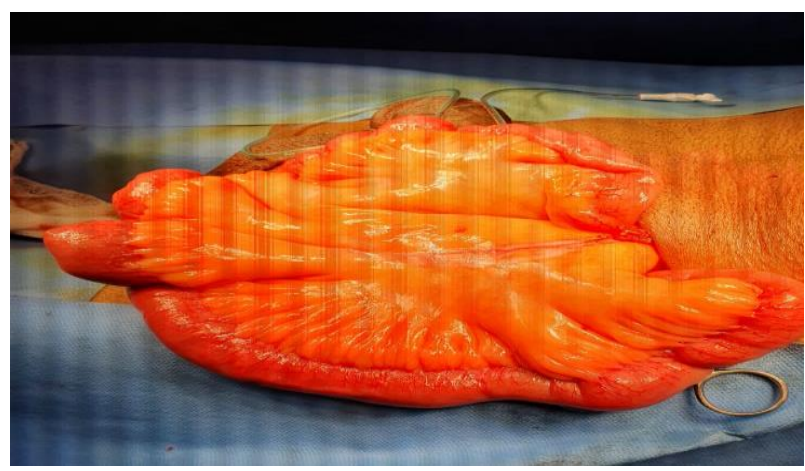

Figure 3: Intraoperative findings, contents of the hernia sac.

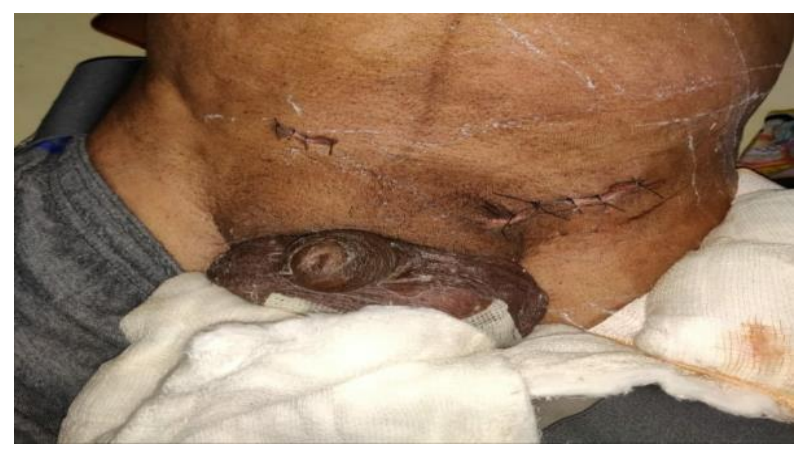

Figure 4: Reduction of left hernia contents within abdominal cavity, postoperative photograph at day 3 .

\section{DISCUSSION}

Giant inguino-scrotal hernias are rare. These are potential source of mental and physical distress to the patient. Giant hernia affects basic functionality, interferes with mobility and activities of daily living, causes pressure effects and may result into urinary and intestinal obstruction, penis may buried inside the scrotum causes dribbling of urine, and due to constant unhygienic skin conditions leads to skin ulceration, excoriation and secondary infection.8,9

Giant inguinal hernias are classified into three types by Trakarnsagna et al.10

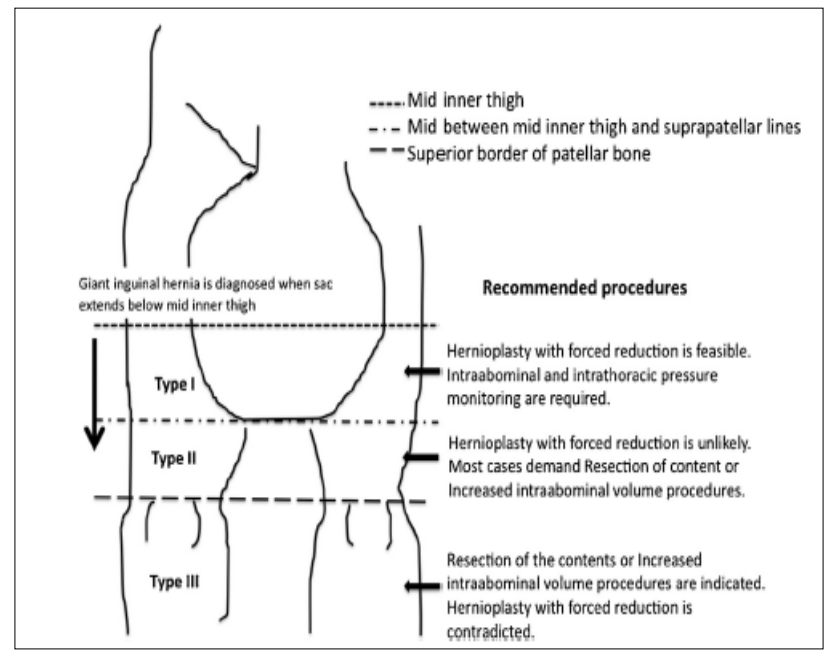

Figure 4: New classification of giant inguinal hernia and recommended procedure.

Due to long term herniation of abdominal viscera, abdomen loses its tone and accustomed to being empty, phenomenon is called loss of domain. ${ }^{11-13}$ Reduction of the hernia disturbs this adapted equilibrium and may results into complications which are associated with high mortality and morbidity.

According to previous literatures, hernioplasty with forceful reduction is feasible only for the type I or mild inguinal hernias, which extends below mid inner thigh but above an imaginary line at lower thigh, the line between the middle point of inner thigh and suprapatellar. After the reduction, close monitoring of intraabdominal and intra thoracic pressure is necessary. This technique may not be appropriate for moderate and severely enlarged giant inguinal hernia (type II and type III). Resection or debulking of the content (omentum, small bowel, and colon) is an option to prevent intra-abdominal hypertension but anastomotic site leak, change in bowel functions and intra-abdominal infections are major limitations of the surgery.

Several other techniques were developed to avoid bowel resection like preoperative progressive pneumoperitoneum and lengthening of abdominal wall by rotation of viable tissue. 
In pre-operative progressive pneumoperitoneum technique, a progressive pneumoperitoneum is created pre-operatively, to ensure the adequate room before the reduction of hernia contents, by gradually insufflating the gas into abdominal cavity via a catheter in situ over 7-14 days. Contraindication being abdominal infections, cardiac conditions and small neck of hernia Also, prolonged hospitalization, and spread of air into hernia sac is some of the major limitation of this technique. ${ }^{14-18}$ Rotation of viable tissues includes techniques like scrotal skin flaps and tensor fascia lata musculocutanous skin flaps. ${ }^{7,13}$ In scrotal skin flap, anterior midline defect was created to increase the volume/space in abdominal cavity. Then, the hernia orifice and midline defect was repaired by prosthetic mesh and then the midline mesh was covered with musculo-cutaneous scrotal flap. In tensor fascia lata musculocutanous flap technique, tensor fascia lata muscle is used instead of scrotal skin to cover the midline defect after mesh placement. Surgical expertise is required to prevent complications in this technique.

Component separation technique is basically freeing external oblique from internal oblique and advancing the rectus muscle to create room for accommodation of hernial contents into the abdominal cavity. ${ }^{19}$

Ramirez and colleagues in 1990 originally described techniques of fascial advancement (anterior component separation) to aid in definitive reconstruction of abdominal wall. In their component separation, anterior component separation (ACS), Ramirez and colleagues first released the posterior sheath. In $30 \%$ of their patients, this was insufficient to permit midline closure, and they therefore created large skin flaps to expose and release the external oblique muscle. ${ }^{20}$

This technique is well suited to large ventral hernia defects for which primary fascial closure with or without mesh reinforcement could result in excessive tension leading to failure of the hernia repair, abdominal compartment syndrome, or measurable changes in pulmonary function. $^{21}$

Traditional open component separation is an excellent technique for midline musculo-fascial defect repair, but it results in significant subcutaneous dead space and transection of the rectus abdominis myocutaneous perforator vessels that supply the overlying skin. Recently developed minimally invasive component separation with inlay bioprosthetic mesh (MICSIB), technique preserves the integrity of both the rectus abdominis myocutaneous perforator vessels that supply the overlying skin and the connection of the subcutaneous fat to the anterior rectus sheath. ${ }^{22}$

Hamad et al in 2013 performed laparoscopic component separation technique to repair a massive inguinal hernia. Technique was proven to be good surgical alternative to address the problem of loss of domain in patients with less morbidity and mortality while it requires a highly skilled professionals and multi-disciplinary approach. ${ }^{3}$

In 2018, Sanford et al treated a giant inguinoscrotal hernia with loss of domain by minimally invasive multidisciplinary approach. 4 weeks before the surgery botulinum toxin injections were given in abdominal muscle for relaxation then, progressive pneumoperitoneum was created followed by eTEP in adjunct with TAR release to create a large space from subcostal down to the myopectineal line. Scrotectomy and scrotoplasty by using adjacent tissue transfer technique completing the reconstruction was done in the end..$^{23}$

\section{CONCLUSION}

Giant inguinal hernia is rare condition defined as hernia sac extends below mid inner thigh in standing position. Surgical repairs are challenging and are associated with high morbidity and mortality due to increase in intraabdominal pressure. Minimal invasive anterior component separation increases the intra-abdominal volume by using tunnel incisions to release external oblique aponeurosis after reduction of hernia contents and Lichtestein mesh hernioplasty can be easily performed. There are several operative techniques used now a days to manage inguinoscrotal hernias, we believe that our technique is unique, less invasive and less expensive than any other methods. Our technique has added advantage of being single stage and doesn't have complications associated with bowel resections, pneumoperitoneum and skin flaps. We used this technique in patient with left sided type II giant inguinoscrotal hernia with several co-morbidities and have achieved results without any postoperative complications.

\section{Funding: No funding sources Conflict of interest: None declared Ethical approval: Not required}

\section{REFERENCES}

1. Lau WY. History of treatment of groin hernia. World J Surg. 2002;26(6):748-59.

2. Hodgkinson DJ, Mcllrth DC. Scrotal reconstruction for giant inguinal hernia. Surg Clin North Am. 1980;64(2):307-13.

3. Hamad A, Marimuthu K, Mothe B, Hanafy M. Repair of massive inguinal hernia with loss of abdominal domain using laparoscopic component separation technique. J Surg Case Rep. 2013;3.

4. Stoppa RE. The treatment of complicated groin and incisional hernias. World J Surg. 1989;13:545-54.

5. Mendez-Fernandez MA, Hollan C, Frank DH, Fisher JC. The scrotal myocutaneous flap. Plast Reconstr Surg. 1986;78(5):676-8.

6. Serpell JW, Polglase AL, Antsee EJ. Giant inguinal hernia. Aust NZJ Surg. 1988;58(10):831-4. 
7. Mehendal FV, Taams KO, Kingsnorth AN. Repair of a giant inguinoscrotal hernia. Br J Plast Surg. 2000;53(6):525-9.

8. Dinesh HN, Kumar J, Shreyas N. Giant inguinoscrotal hernia repaired by Lichtensteins technique without loss of domain- a case report. J Clin Diagn Res. 2014;8(9):7-8.

9. Karthikeyan VS, Sistla SC, Ram D, Ali SM, Rajkumar N. Giant inguinoscrotal hernia- report of a rare case with literature review. Int Surg. 2014;99(5):560-4.

10. Trakarnsagna A, Chinswangwatanakul V, Methasate A, Swangsri J, Phalanusitthepha C, Parakonthun T et al. Giant inguinal hernia: Report of a case and reviews of surgical techniques. Int J Surg Case Rep. 2014;5(11):868-72.

11. Forrest J. Repair of massive inguinal hernia. Arch Surg. 1979;114:1087-8.

12. Moreno IG. The rational treatment of hernias and voluminous chronic eventrations: preparation with progressive pneumoperitoneum. In: Nhylus LM, Condon RE, eds. Hernia, 2nd edn. Philadelphia: J.B. Lippincott Co. 1978;536-50.

13. Merrett ND, Waterworth MW, Green MF. Repair of giant inguinoscrotal inguinal hernia using marlex mesh and scrotal skin flaps. Aust N Z J Surg. 1994;64(5):380-3.

14. Moreno IG. Chronic eventrations and large hernias; preoperative treatment by progressive penumoperitoneum; original procedure. Surgery. 1947;22(6):945-53.

15. Cannolly DP, Perri FR. Giant hernias managed by pneumoperitoneum. JAMA. 1969;209(1):71-4.

16. Barst HH. Pneumoperitoneum as an aid in the surgical treatment of giant herniae. Br J Surg. 1972;59(5):360-4.

17. Mayagoitia JC, Suarez D, Arenas JC, Diaz de Leon V. Preoperative progressive pneumoperitoneum in patients with abdominal wall hernias. Hernia. 2006;10(3):213-7.

18. Piskin T, Aydin C, Barut B, Dirican A, Kayaalp C. Preoperative progressive peumoperitoneum for giant inguinal hernias. Ann Saudi Med. 2010;30(4):31720.

19. Valliattu AJ, Kingsnorth AN. Single-stage repair of giant inguinoscrotal hernia using the abdominal wall component separation technique. Hernia. 2008;12(3):329-30.

20. Ramirez OM, Ruas E, Dellon AL. "Component separation" methods for closure of abdominal wall defects: an anatomical and clinical study. Plast Reconstr Surg. 1990;86:519-26.

21. Agnew SP, Small W, Wang E, Smith LJ, Hadad I, Dumanian GA. Prospective measurements of intraabdominal volume and pulmonary function after repair of massive ventral hernias with the components separation technique. Ann Surg. 2010;251(5):981-8.

22. Butler CE, Campbell KT. Minimally invasive component separation with inlay bioprosthetic mesh (MICSIB) for complex abdominal wall reconstruction. Plast Reconstr Surg. 2011;128(3):698-709.

23. Sanford Z, Weltz AS, Singh D, Hanley R, Todd D, Belyansky I. Minimally Invasive Multidisciplinary Approach to Chronic Giant Inguinoscrotal Hernias. Surg Innov. 2019;26(4):427-31.

Cite this article as: Sewalia P, Chawla AS, Sangtam LT, Mandalapu H, Kumar H, Kaur A, et al. Repair of giant inguino-scrotal hernia with loss of domain using minimally invasive anterior component separation technique combined with Lichtenstein tension-free mesh hernioplasty. Int Surg J 2021;8:406-10. 